\title{
Capturing the Fantastic Voyage of Monocytes Through Time and Space
}

\author{
Ye Chean Teh ${ }^{1,2}$, Jeak Ling Ding ${ }^{2}$, Lai Guan $\mathrm{Ng}^{1,3,4}$ and Shu Zhen Chong ${ }^{1 *}$ \\ ${ }^{1}$ Functional Immune Imaging, Singapore Immunology Network (SIgN), A*STAR (Agency for Science, Technology and \\ Research), Biopolis, Singapore, ${ }^{2}$ Department of Biological Sciences, National University of Singapore (NUS), Singapore, \\ Singapore, ${ }^{3}$ Department of Microbiology \& Immunology, Immunology Programme, Life Science Institute, Yong Loo Lin \\ School of Medicine, National University of Singapore, Singapore, Singapore, ${ }^{4}$ School of Biological Sciences, Nanyang \\ Technological University, Singapore, Singapore
}

Monocytes are a subset of cells that are categorized together with dendritic cells (DCs) and macrophages in the mononuclear phagocyte system (MPS). Despite sharing several phenotypic and functional characteristics with MPS cells, monocytes are unique cells with the ability to function as both precursor and effector cells in their own right. Before the development of hematopoietic stem cells (HSCs) in utero, monocytes are derived from erythro-myeloid precursors (EMPs) in the fetal liver that are important for populating the majority of tissue resident macrophages. After birth, monocytes arise from bone marrow (BM)-derived HSCs and are released into the circulation upon their maturation, where they survey peripheral tissues and maintain endothelial integrity. Upon sensing of microbial breaches or inflammatory stimuli, monocytes migrate into tissues where their plasticity allows them to differentiate into cells that resemble macrophages or DCs according to the environmental niche. Alternatively, they may also migrate into tissues in the absence of inflammation and remain in an undifferentiated state where they perform homeostatic roles. As monocytes are typically on the move, the availability of intravital imaging approaches has provided further insights into their trafficking patterns in distinct tissue compartments. In this review, we outline the importance of understanding their functional behavior in the context of tissue compartments, and how these studies may contribute towards improved vaccine and future therapeutic strategies.

Keywords: monocytes, marginal pool, bone marrow, spleen, CXCR4 $=$ chemokine receptor 4 , inflammation, steady-state, intravital 2P microscopy

\section{INTRODUCTION}

When agent Grant was traveling through the blood vessels of Dr Jan Benes in the science fiction movie "Fantastic Voyage," he might have noticed a large white blood cell with abundant cytoplasm and a hefty eccentrically placed kidney bean-shaped nucleus. This cell measured approximately $20 \mu \mathrm{m}$ in diameter and was the largest of all circulating leukocytes. Known as the monocyte, this cell is renowned for its phagocytic activity and constitutes about $5-10 \%$ of total blood leukocytes.

For half a century, monocytes were touted to be an intermediate cell type with the sole purpose of replenishing tissue macrophages $(1,2)$. This dogma was based on Van Furth and Cohen's findings in the mid twentieth century $(3,4)$ and has been a subject of intense research and debate in the past decade. While genetic fate-mapping experiments have since revealed embryonic progenitors as the precursors of most tissue macrophages (5-7), it is increasingly apparent that these original 
theories are not entirely incorrect either. Instead, it is now proposed that monocytes have the ability to reconstitute the macrophage pool, in a temporal and spatial manner $(8,9)$, with competition for a restricted number of niches as the main driving factor (10).

With monocytes no longer functioning solely as steadystate macrophage precursors, it remains unclear what tasks they may perform in immunity and host defense. Monocytes are heterogeneous and consist of a classical population $\left(\mathrm{Ly} 6 \mathrm{C}^{\text {hi }}\right.$ in mice; $\mathrm{CD} 14^{++} \mathrm{CD} 16^{-}$in humans) and a non-classical population (Ly6 $\mathrm{C}^{\text {lo }}$ in mice; $\mathrm{CD} 14^{+} \mathrm{CD} 16^{+}$in humans) $(7,11$, 12) with distinct functional roles (13). Interestingly, amidst the flurry of excitement in examining macrophage ontogeny by genomics/epigenomics approaches, the understanding of monocyte function in the context of spatial distribution and tissue niche was also steadily emerging as a key focus area. Together with the development in molecular and cell biological studies (14), the advent of imaging techniques such as twophoton intravital microscopy (2P-IVM), which allows direct visualization of immune cells using fluorescent reporter-tagged mice in vivo and in situ $(15,16)$, has helped to uncover a wide array of imperative monocyte biology. Nevertheless, monocyte behavior is highly distinct in each tissue compartment due to their plasticity and sensitivity to niche signals (17). Therefore, it is extremely vital that we consider their functional role in a dynamic and spatiotemporal manner. In this mini-review, we will provide insights on the trafficking patterns of monocytes and how their behavior in distinct tissue compartments governs their function in immune responses (Figure 1).

\section{TRAVELING BACK IN TIME: RECOGNITION OF THE FETAL MONOCYTE}

When van Furth and Cohen's proposal of ontogeny of tissue macrophages arising solely from monocytes (3) was challenged in the early twenty-first century, scientists postulated that adult tissue macrophages were derived from embryonic precursors before birth instead $(6,7,18)$. In mice, these embryonic precursors emerged before the development of hematopoietic stem cell (HSC) progenitors and comprised of erythro-myeloid precursors (EMPs) that appear in the yolk-sac blood islands of the embryo at around E7.0 of gestation $(19,20)$. Importantly, these EMPs could bypass the monocyte stage and give rise directly to primitive macrophages that would seed the organs of the growing embryo $(6,21,22)$. However, it was later discovered that upon establishment of the blood circulation, these EMPs migrate and seed the fetal liver at E9.5 of gestation (19, 23, 24), giving rise to multiple myeloid lineage cells, including a very important cell type-the fetal monocyte (25-27).

\footnotetext{
Abbreviations: BM, bone marrow; cMop, common monocyte progenitor; CSF-1, colony stimulating factor-1; DC, dendritic cell; EMP, erythro-myeloid precursor; HSC, hematopoietic stem cell; IL, interleukin; iNOS, inducible nitric oxide synthase; LPS, lipopolysaccharide; MHC, major histocompatibility complex; PLVAP, plasmalemma vesicle-associated protein; S1PR5, sphingosine-1-phosphate receptor 5; 2P-IVM, two-photon intravital imaging; TNF, tumor necrosis factor; TpMo, transitional pre-monocyte; ZT, Zeitgeber.
}

In mice, fetal monocytes were first reported by Naito et al. and were shown to emerge in the fetal liver around E12.5 before being released into the blood from E13.5 onwards $(27,28)$. Despite primitive macrophages already occupying the tissue niches at this stage, fetal monocytes were discovered to colonize the remaining open niches of every tissue at E14.5 with the exception of the brain $(26,29-32)$ (Figure 1A). To date, little is known about the trafficking mechanisms that are adopted by fetal monocytes. Nevertheless, fetal monocyte migration into tissues is independent of the CCR2-CCL2 axis (26) while their egress from the fetal liver is dependent on plasmalemma vesicle-associated protein (PLVAP), which is an endotheliumspecific molecule that forms diaphragm-like structures in the fenestrae of the liver sinusoidal endothelium (33) (Figure 1A). Functionally, fetal monocytes share many common traits with adult BM-derived monocytes but have reduced expression of antigen presentation and pathogen recognition-associated genes (26). In contrast to adult monocytes, fetal monocytes also retain a high proliferative capacity in tissues that is CSF-1 receptor independent (29), thereby allowing fetal monocytes to harbor a competitive advantage in replenishing tissue macrophages (34). Further investigations would be required to comprehend how fetal monocytes traffic into tissues and what signals affect their retention in their respective niches as they differentiate into macrophages.

\section{MONOCYTES IN-WAITING: THE BONE MARROW AND SPLEEN}

Unlike fetal monocytes that are derived from late EMPs in the fetal liver, adult monocytes originate from HSC progenitors in the $\mathrm{BM}$ after birth $(7,35,36)$. It was initially thought that Ly6C ${ }^{\text {hi }}$ monocytes originated directly from the common monocyte progenitor (cMop) and are poised to leave the $\mathrm{BM}$ upon maturing beyond the cMop stage (35). However, contrary to this assumption, recent findings by Chong et al. have demonstrated that cMops undergo an additional step of maturation into a transitional precursor before the ensuing mature monocytes (37). This transitional precursor was termed "transitional pre-monocytes" (TpMos), and was discovered when BM Ly6C $\mathrm{C}^{\text {hi }}$ monocytes were found to contain two distinct subpopulations: (1) the CXCR $4{ }^{\text {hi }}$ subpopulation, which constitutes TpMos derived directly from cMops and are immobilized in the $\mathrm{BM}$ where they proliferate rapidly to replenish mature monocytes; (2) the $\mathrm{CXCR} 4^{\text {lo }}$ subpopulation, which consists of bona fide mature Ly6C hi monocytes that have exited the cell cycle and are readily mobilized from the BM (37) (Figure 1Bi). Since TpMos are highly proliferative and immobilized in the BM under regular circumstances, their presence likely serves as a regulatory checkpoint for the rapid replenishment and prevention of an uncontrolled release of BM monocytes.

In comparison to other myeloid cells (38), monocytes transit quickly through the $\mathrm{BM}$ and are released rapidly into the circulation after their last division (39). Their egress and retention in the $\mathrm{BM}$ is critically dependent on CCR2-signaling 


\section{A Before Birth}

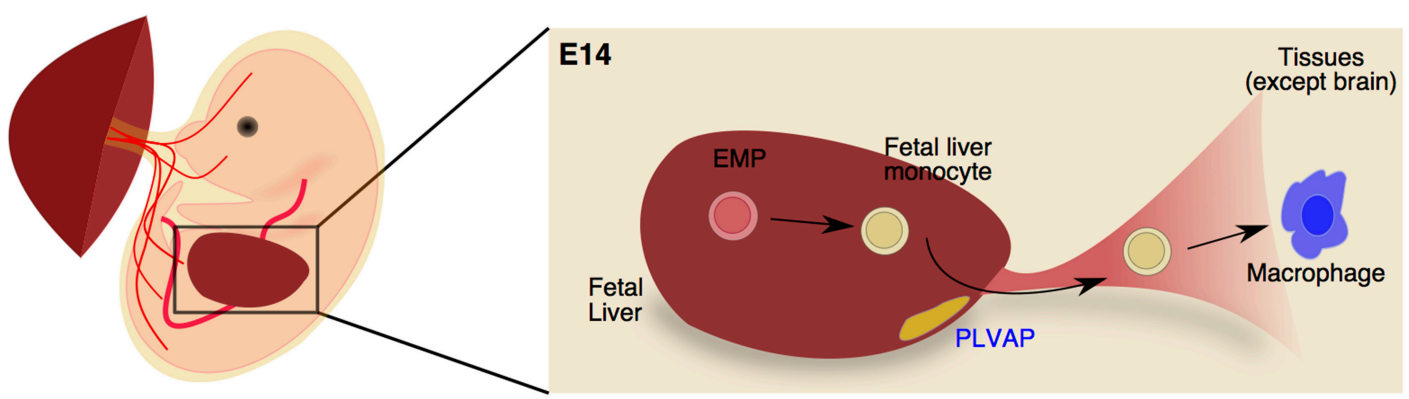

\section{B After Birth}

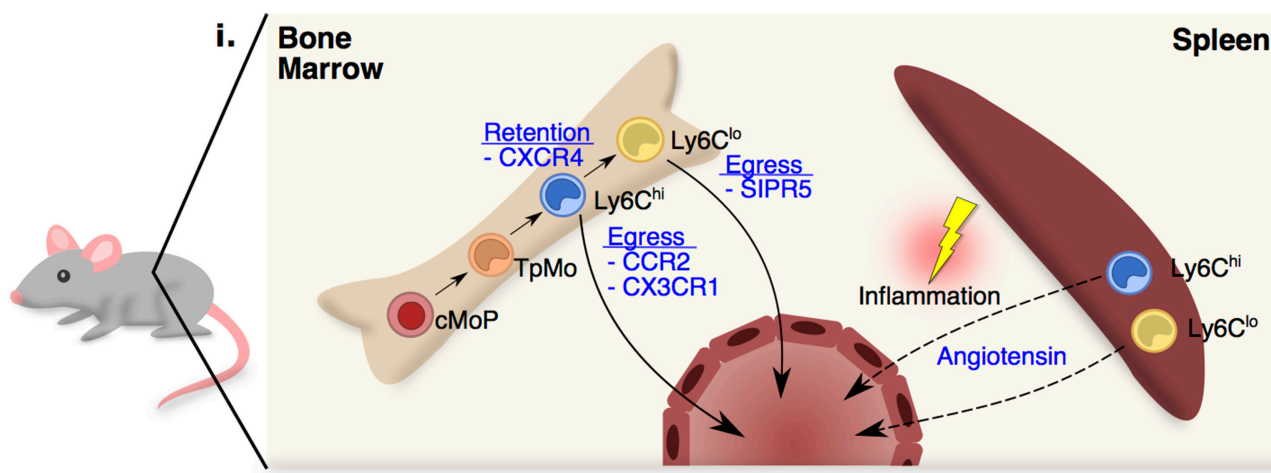

\section{Reservoir}

ii.

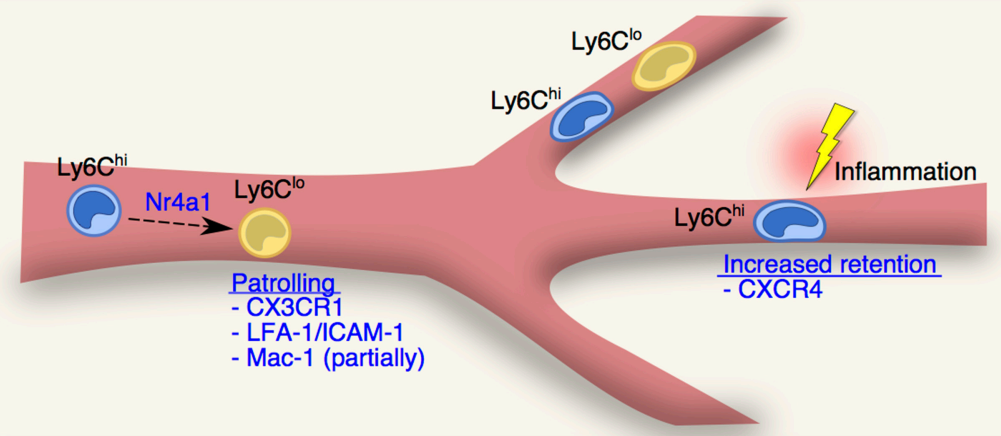

iii.
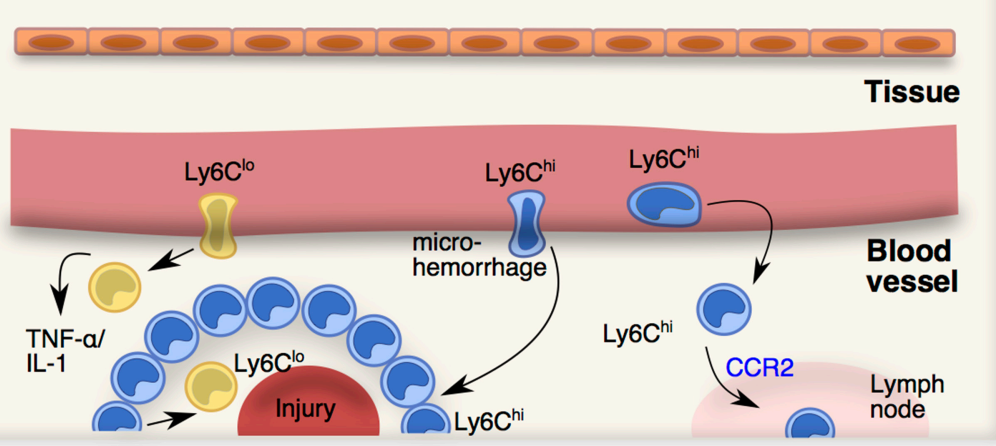

\section{Marginal \\ pool}

Tissue

FIGURE 1 | Monocyte trafficking and function in distinct stages and peripheral sites. (A) From E13.5 onwards, fetal monocytes derived from erythro-myeloid precursors (EMPs) in the fetal liver can be released into the circulation in a plasmalemma vesicle-associated protein (PLVAP) dependent manner. At E14.5, these fetal monocytes will colonize the open niches of every tissues as fetal monocyte-derived macrophages except the brain. (B) After birth (i) Adult monocytes originate from the common monocyte progenitors (cMoPs) that give rise to Ly6 $\mathrm{C}^{\mathrm{hi}}$ monocytes through a transitional precursor called transitional pre-monocytes (TpMos). Ly6 $\mathrm{C}^{\mathrm{hi}}$ 
FIGURE 1 | monocytes are released into the circulation upon their last division, and differentiate into Ly6Clo monocytes. The retention and egress of Ly6Chi monocytes are dependent on CXCR4- and CCR2-signaling respectively, whereas Ly6Clo monocytes egress is dependent on S1PR5-signaling. At steady state, circulating monocytes enter the spleen as a secondary reservoir. During inflammation, splenic Ly6C ${ }^{\text {hi }}$ and Ly6Clo monocytes are mobilized into the circulation via Angiotensin-II/AGTR1A-signaling. (ii) Upon entering the circulation, short-lived Ly6 $\mathrm{C}^{\text {hi }}$ monocytes gradually differentiate into longer-lived Ly6Clo monocytes via Nr4a1-signaling. Ly6Clo monocytes patrol the vessels partially via Mac-1, but significantly via CX3CR1-signaling and LFA-1/ICAM-1 interaction with the endothelial cells. At steady state, Ly6Chi monocytes do not interact closely with the endothelium except in the vascular beds of distinct peripheral organs. CXCR4 regulates steady state monocyte margination in the lung. During inflammation, Ly6C ${ }^{\text {hi }}$ monocytes increased their transit time, resulting in increased retention in the microvasculature. (iii) At steady state, Ly6 $C^{\text {hi }}$ monocytes survey the tissue environment for antigens to transport into draining lymph nodes. During injury, Ly6C ${ }^{l o}$ monocytes infiltrate rapidly into inflamed site to provide TNF- $\alpha$ and IL-1. Besides the classical rolling and migration steps, a proportion of Ly6Chi monocytes utilizes microhemorrhages to extravasate and enter inflammatory sites rapidly and form a ring-like structure before differentiating into Ly6Clo monocytes for tissue repair.

(40,41) and CXCR4-signaling (37, 42, 43), respectively. Unlike vascular monocytes that are highly motile, Ly $6 \mathrm{C}^{\text {hi }}$ monocytes in the $\mathrm{BM}$ parenchyma are comparatively sessile, displaying slow random displacements (44) while being juxtaposed to $\mathrm{Nestin}^{+}$stromal cells $(42,45)$. Upon sensing of inflammatory stimuli like LPS through Toll-like receptor 4 (45), Nestin ${ }^{+}$ stromal cells express CCL2 (42), which causes BM Ly6C ${ }^{\text {hi }}$ monocytes to increase their velocity and displacement (46). This CCL2 exposure also leads to desensitization of monocyte response to CXCL12 (ligand of CXCR4) possibly through internalization of CCR2-CXCR4 complexes, which weakens the CXCR4 anchoring signal and results in their eventual egress (42). Furthermore, only mature Ly6 $\mathrm{C}^{\text {hi }}$ monocytes, and not TpMos, were able to leave the BM under subclinical doses of LPS because TpMos were unable to respond to CCL2 as efficiently as mature Ly6C ${ }^{\text {hi }}$ monocytes (37). CX3CR1 was also discovered to regulate $L y 6 C^{\text {hi }}$ monocyte numbers in the BM after cyclophosphamide-induced myeloablation although their effect is less pronounced than CCR2-signaling (47). While signals governing the release of BM Ly6Chi monocytes are well-documented, mechanisms regulating Ly6 $\mathrm{C}^{\text {lo }}$ monocyte egress are less defined. Nevertheless, it was discovered that Ly6C $\mathrm{C}^{\mathrm{lo}}$ monocytes have very low levels of the CCR2 receptor and thus their egress is more likely to depend on S1PR5 (48).

Besides the BM, monocytes have also been found to reside in the subcapsular red pulp of the spleen as a secondary reservoir (49). In contrast to the $\mathrm{BM}$ whose main function lies in immune cell generation from HSC progenitors, the spleen functions mainly as a lymphatic organ (50). Therefore, the steady state monocyte reservoir is not generated in the spleen itself, but derived from circulating monocytes that have entered the spleen (49). Exceptions to this rule, however, do occur in the case of extramedullary hematopoiesis, when monocyte progenitors were found to expand in the spleen during inflammation, contributing to the monocyte reservoir in situ $(51,52)$. More importantly, splenic monocytes can increase their motility and exit into the blood during myocardial infarction via Angiotensin II-signaling and this process is independent of CCR2-signaling (49) (Figure 1Bi). Interestingly, Angiotensin IIdependent recruitment of monocytes into the infarct (a localized area of dead tissue resulting from failure of blood supply) is strictly mediated from the spleen and peripheral circulation, but not from the BM (51). Splenic monocytes were also found to be mobilized to the ovaries where they enhance ovulatory processes (53). Notably, the spleen is also a key site for an alternative source of monocytes in cardiovascular diseases (52, 54, 55), tumor progression (56) and lung ischemia (57). These findings hence suggest that the spleen fulfills the urgent demand of monocytes during inflammation by providing an emergency source, which extends time for the BM to generate more monocytes concurrently.

\section{MONOCYTES ON-THE-GO: NAVIGATING THROUGH THE CIRCULATORY HIGHWAYS}

Upon entering the circulation, monocytes rely heavily on the circulatory system for transportation to peripheral compartments. Ly6C $\mathrm{C}^{\text {hi }}$ monocytes have a half-life of approximately $20-24 \mathrm{~h}$ in the peripheral blood before gradually differentiating into Ly6C $\mathrm{C}^{\mathrm{lo}}$ monocytes (half-life of $48 \mathrm{~h}$ in mice; 7 days in humans) via Nr4a1-signaling (58-61). Unlike classical Ly6Chi monocytes that roll along vessels, CX3CR1 high non-classical Ly6C ${ }^{\text {lo }}$ monocytes in mice (62) and their human counterparts (CD14 ${ }^{+} \mathrm{CD} 16^{+}$monocytes) (63) patrol vessels by crawling at a speed of $12 \mu \mathrm{m} / \mathrm{min}$. Their patrolling behavior is partially mediated by Mac-1 and is highly dependent on CX3CR1-signaling and LFA-1/ICAM-1 or ICAM2 interaction with endothelial cells $(62,64,65)$. Furthermore, this patrolling activity is critical for micro-scavenging the luminal surface of vessels and maintaining endothelial integrity (64) (Figure 1Bii). Notably, an increase in atherosclerotic endothelial apoptosis (66), amyloid deposition (67) and tumor metastasis (68) was observed when Ly6 $\mathrm{C}^{\mathrm{lo}}$ monocytes were absent in $\mathrm{Nr}_{4} \mathrm{al}^{-/-}$mice. Because of their close interaction with vessels, Ly6 $\mathrm{C}^{\text {lo }}$ monocytes orchestrate the recruitment and activation of neutrophils upon sensing a breach in vascular integrity through TLR7signaling, which subsequently leads to their retention in the capillaries $(64,69)$.

In contrast to $\mathrm{Ly} 6 \mathrm{C}^{\mathrm{lo}}$ monocytes that patrol vessels, it is commonly recognized that Ly6 $\mathrm{C}^{\text {hi }}$ monocytes do not interact closely with the endothelium in the steady-state (70). However, exceptions to this rule do occur in vascular beds of distinct peripheral organs. These vascular beds consist of multiple smallcaliber microvessels $(<5 \mu \mathrm{m}$ in diameter), which necessitate larger leukocytes $(6-8 \mu \mathrm{m})$ to deform and physically interact with the endothelium for their transit (71). This phenomenon results in substantial leukocyte retention and the formation of a "marginal pool." In particular, the lungs represent a major site of leukocyte margination, and classical Ly6 $\mathrm{C}^{\text {hi }}$ monocytes were discovered to form close interactions with the lung vasculature 
under resting state $(37,72,73)$. Ly6C hi monocytes are highly adherent upon contact with surfaces and can be seen to extend their pseudopods upon movement (Figure 2A). Notably, we discovered that CXCR4 regulates steady-state monocyte margination in the lung (37) (Figure 1Bii). Upon endotoxin sensing, classical Ly6 $\mathrm{C}^{\text {hi }}$ monocytes increased their lung transit time (74) by adhering to the endothelium, resulting in increased predisposition towards lung injury that can be reversed with CXCR4 inhibition (37). Apart from the lung, intravital imaging of monocytes in vascular beds of the kidney $(75,76)$ and liver (77) revealed increased retention of monocytes in the microvasculature during inflammation. Increased adhesion of Ly6C hi monocytes, but not neutrophils, in the brain microvasculature during cerebral malaria is also associated with progressive worsening of clinical symptoms (78). Additionally, the BM was discovered to contain a CX3CR1-dependent marginal pool of monocytes that can be rapidly deployed to the peritoneum (79).

Since the BM is constantly releasing monocytes into the circulation, it is conceivable that a counterbalancing mechanism exists to ensure that circulating monocyte numbers return to homeostasis. Indeed, CXCR4-signaling keeps this homeostasis in check by influencing the spatiotemporal localization of monocytes between the circulation and peripheral compartments (Figure 2B). Notably, circulating monocytes were found to return at a constant rate to the $\mathrm{BM}$ and spleen parenchyma in a CXCR4-dependent manner (37). More importantly, the number of circulating monocytes compared to the numbers in the peripheral compartments were found to vary according to circadian rhythmic oscillations, with more monocytes present in the circulation at Zeitgeber 5 (ZT5) than ZT13 in mice (where ZT0 refers to lights on and ZT12 to lights off) $(37,80)$. This diurnal oscillation of monocyte numbers is regulated by the circadian gene, Bmal (80), and also corresponds with diurnal fluctuations in CXCR4 levels on mature monocytes (37), such that absence of CXCR4 also abolishes the diurnal oscillation in monocyte numbers.

\section{MONOCYTES EXITING THE HIGHWAYS: EXPLORING TISSUES}

The entry of monocytes into tissues is critical for pathogen clearance and wound healing. Furthermore, it is typically acknowledged that their time of entry dictates their function, as ingress of monocytes in the early phase of inflammation is associated with a pro-inflammatory phenotype, while their presence in the later phase corresponds to an antiinflammatory function $(81,82)$ (Figure 1Biii). Mediators that attract circulating monocytes into tissues include chemokines, complement components, and products of tissue matrix degradation (83). Since patrolling Ly6 $\mathrm{C}^{\text {lo }}$ monocytes interact closer with the endothelium compared to Ly6 $\mathrm{C}^{\text {hi }}$ monocytes, it is conceived that their migratory dynamics into tissues are quicker than $\mathrm{Ly} 6 \mathrm{C}^{\text {hi }}$ monocytes. Indeed, Ly6C $\mathrm{C}^{\text {lo }}$ monocytes infiltrate within an hour into inflamed tissues induced by aseptic wounding, irritants or Listeria monocytogenes to provide the initial sources of TNF- $\alpha$ and IL-1 (62). In contrast, Ly6C ${ }^{\text {hi }}$ monocyte recruitment into tissues typically occurs $24-48 \mathrm{~h}$ after injury (84). Their entry into tissues involves vascular rolling, adhesion, and transendothelial migration that has been welldocumented $(14,83,85)$. Nevertheless, a proportion of Ly6C ${ }^{\text {hi }}$ monocytes have also been shown to utilize microhemorrhages to exit blood vessels and enter inflammatory sites rapidly (86). This allows Ly6Chi monocytes to enter the injury site as quickly as neutrophils, where they were found to scout the wound bed randomly before progressively slowing down over a study period of $2.5 \mathrm{~h}(86)$. While it is unclear what causes this behavioral change, it is likely that this may be associated with the conversion of Ly6 $\mathrm{C}^{\text {hi }}$ into Ly6 $\mathrm{C}^{\text {lo }}$ monocytes that is critical for wound healing. Indeed, $\mathrm{Ly} 6 \mathrm{C}^{\mathrm{hi}}$ monocytes entered the injury site and formed a ring-like structure around the injured foci that persisted for $48 \mathrm{~h}$ in a model of sterile hepatic injury (77). These Ly6C ${ }^{\text {hi }}$ monocytes subsequently differentiated into Ly6 $\mathrm{C}^{\text {lo }}$ monocytes after sensing IL-4 and IL-10 within the ring-like structure. Notably, this phenotypic conversion was critical for monocytes to move further into the injury area and to initiate optimal repair. These findings further highlight the plasticity of monocytes in their functional reprogramming by switching from an inflammatory phenotype to a profile that facilitates wound repair.

Upon entering tissues, infiltrating monocytes progressively alter their phenotype by adopting macrophage characteristics while losing monocyte features, and this gradual differentiation process is known as the classical "monocyte waterfall" effect $(8,87,88)$. Besides replacing certain residential macrophages in the steady-state $(6,18)$, monocytes may also differentiate into TNF/iNOS-producing DCs (Tip-DCs) (89), wound-associated macrophages (WAMs) (90) or tumor-induced myeloid suppressor cells (91). However, bona fide classical monocytes have also been found to remain undifferentiated in the tissue at resting state (92). These monocytes extravasated constitutively into tissues and lymph nodes in a CCR2-dependent manner and retained most of their existing monocyte transcriptional profile. Nevertheless, these Ly6 $\mathrm{C}^{\text {hi }}$ monocytes increased their expression of MHCII, co-stimulatory molecules and CCR7, suggesting that these cells survey the tissue environment for antigens to transport to draining lymph nodes in the steady state. Since monocyte extravasation into tissues in the steady-state was found to be microbiota-independent (92), it would be interesting to determine the specific mechanisms that dictate their migration into tissues and the factors that preserve their profile in these circumstances.

\section{CONCLUSION AND FUTURE PERSPECTIVES}

Despite being described in many important studies in the last century, our comprehension of monocyte biology has only taken a substantial leap in the past decade upon the advent of highly sophisticated imaging techniques that complement the current use of biochemistry, cell biology and genetic tools. More importantly, 2P-IVM has unveiled critical trafficking 
A i.

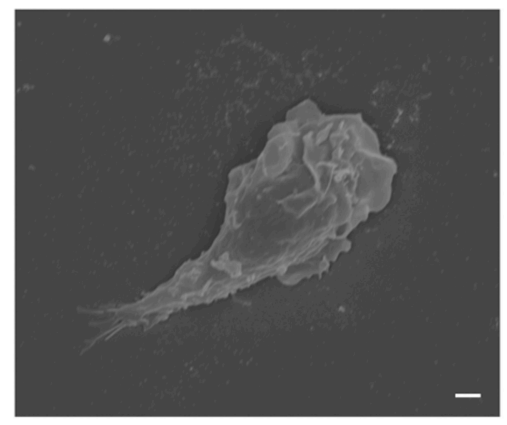

ii.

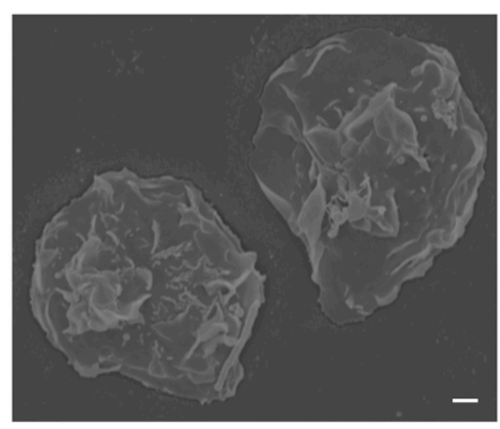

B

Bone marrow

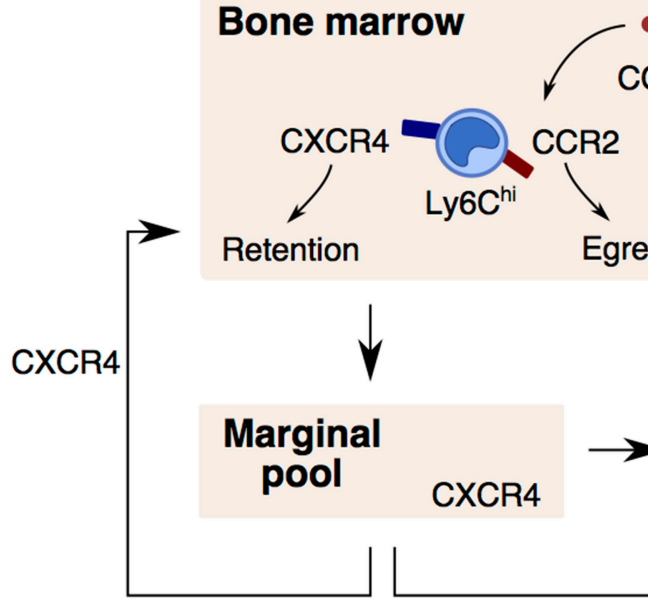

Bma1 gene ZT5 CXCR4 $\downarrow$ ZT13 CXCR4 $\uparrow$
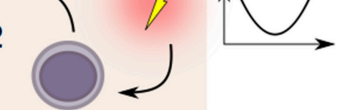
Stromal cell

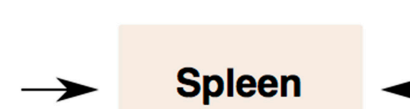

$\rightarrow \quad$ Spleen

Microbiotaindependent

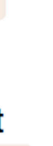

Tissues

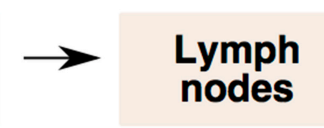

FIGURE 2 | CXCR4 controls monocyte trafficking into different peripheral compartments. (A) Scanning electron microscopy images of a Ly6C hi monocyte (i) protruding its pseudopod upon adhering to coverslip and (ii) extending their cytoplasmic membrane when fully adhered to the coverslip. Bars, $1 \mu \mathrm{m}$. (B) Monocyte egress and retention in the bone marrow is dependent on CCR2-signaling and CXCR4-signaling. Upon sensing inflammatory stimuli, stromal cells release CCL2, desensitizing monocyte response to CXCL12 (CXCR4 ligand), resulting in monocyte entry into the circulation and spleen. In the circulation, CXCR4 regulates steady-state monocyte margination in tissue marginal pools. Monocytes may also extravasate into tissues and lymph nodes in a microbiota-independent manner. CXCR4-signaling also regulates the homing of circulating monocytes back to the bone marrow and spleen. Monocyte numbers display diurnal oscillation that is regulated by the circadian gene, Bma1. Lower CXCR4 levels at ZT5 (lights on period) results in more circulating monocytes, whereas higher CXCR4 levels at ZT13 (lights off period) results in higher monocyte retention in the bone marrow.

mechanisms that may have important implications for future vaccine designs/therapeutic strategies. In particular, the specific kinetics of monocyte trafficking in different tissue compartments and their interaction with other immune cells will allow scientists to optimize their drug administration and design according to these dynamics. For example, clinicians who aim to reduce tissue inflammation may take advantage of the knowledge that non-classical monocytes recruit neutrophils in the early stages of inflammation (64). Therefore, selecting specific drugs that target molecules only on non-classical monocytes, instead of both monocyte subsets, may help to reduce the likelihood of any off-target effects and secondary infections during long periods of therapy. While 2P-IVM has provided valuable insight, major technical bottlenecks still exist against gaining a global understanding of these cells in chronic disease states. These issues are due to the highly plastic nature of monocytes, which may include the loss of fluorescence signal as they differentiate into monocyte-derived cells. Furthermore, their differentiated phenotypes are distinct in various chronic disease settings
$(13,93)$. In this regard, a combination of tools that would enable researchers to identify monocyte-derived cells with greater spatiotemporal specificity would be beneficial in addressing these issues. In particular, multiplex immunofluorescence techniques $(94,95)$ in a histo-cytometry setting $(96,97)$ that involves optically cleared large tissue samples (98) would provide a global view of their localization and interaction with other immune cells. Furthermore, refining image analysis methods that deal with large volumes of data, such as using a huesaturation-brightness-based surface creation to streamline multichannel image cytometry for three-dimensional images (99), would allow us to uncover new markers on monocyte-derived cells that can be used to generate improved fluorescent-tagged mice. Importantly, while transcriptomic studies have shown mouse and human monocytes to be homologous, a reverse pattern in certain genes such as TREM-1, CD36, CXCR4, and CD9 was also discovered (12). Therefore, future work adopting humanized mice for 2P-IVM studies, is warranted to verify if trafficking mechanisms of mouse monocytes are similar to that 
in humans. Taken together, we believe that the combination of these state-of-the-art imaging tools in future studies will provide further insight into the temporal and spatial landscape of monocytes that could hold the key for future biomarker and therapeutic discoveries.

\section{AUTHOR CONTRIBUTIONS}

YCT, JLD, LGN and SZC wrote and conceptualized the manuscript. YCT did the figures.

\section{REFERENCES}

1. Segura E, Amigorena S. Inflammatory dendritic cells in mice and humans. Trends Immunol. (2013) 34:440-5. doi: 10.1016/j.it.2013.06.001

2. Varol C, Mildner A, Jung S. Macrophages: development and tissue specialization. Annu Rev Immunol. (2015) 33:643-75. doi: 10.1146/annurev-immunol-032414-112220

3. van Furth R, Cohn ZA. The origin and kinetics of mononuclear phagocytes. $J$ Exp Med. (1968) 128:415-35. doi: 10.1084/jem.128.3.415

4. van Furth R, Cohn ZA, Hirsch JG, Humphrey JH, Spector WG, Langevoort HL. The mononuclear phagocyte system: a new classification of macrophages, monocytes, and their precursor cells. Bull World Health Organ. (1972) 46:84552.

5. Hopkinson-Woolley J, Hughes D, Gordon S, Martin P. Macrophage recruitment during limb development and wound healing in the embryonic and foetal mouse. J Cell Sci. (1994) 107:1159-67.

6. Ginhoux F, Guilliams M. Tissue-resident macrophage ontogeny and homeostasis. Immunity. (2016) 44:439-49. doi: 10.1016/j.immuni.2016.02.024

7. Ginhoux F, Jung S. Monocytes and macrophages: developmental pathways and tissue homeostasis. Nat Rev Immunol. (2014) 14:392-404. doi: $10.1038 /$ nri3671

8. Bain CC, Bravo-Blas A, Scott CL, Perdiguero EG, Geissmann F, Henri S, et al. Constant replenishment from circulating monocytes maintains the macrophage pool in the intestine of adult mice. Nat Immunol. (2014) 15:92937. doi: 10.1038/ni.2967

9. Bain CC, Hawley CA, Garner H, Scott CL, Schridde A, Steers NJ, et al. Long-lived self-renewing bone marrow-derived macrophages displace embryo-derived cells to inhabit adult serous cavities. Nat Commun. (2016) 7:ncomms11852. doi: 10.1038/ncomms11852

10. Guilliams M, Scott CL. Does niche competition determine the origin of tissue-resident macrophages? Nat Rev Immunol. (2017) 17:451-60. doi: $10.1038 /$ nri.2017.42

11. Geissmann F, Jung S, Littman DR. Blood monocytes consist of two principal subsets with distinct migratory properties. Immunity. (2003) 19:71-82. doi: 10.1016/S1074-7613(03)00174-2

12. Ingersoll MA, Spanbroek R, Lottaz C, Gautier EL, Frankenberger M, Hoffmann R, et al. Comparison of gene expression profiles between human and mouse monocyte subsets. Blood. (2010) 115:e10-9. doi: 10.1182/blood-2009-07-235028

13. Mildner A, Yona S, Jung S. A close encounter of the third kind: monocyte-derived cells. Adv Immunol. (2013) 120:69-103. doi: 10.1016/B978-0-12-417028-5.00003-X

14. Gerhardt T, Ley K. Monocyte trafficking across the vessel wall. Cardiovasc Res. (2015) 107:321-30. doi: 10.1093/cvr/cvv147

15. Germain RN, Robey EA, Cahalan MD. A decade of imaging cellular motility and interaction dynamics in the immune system. Science. (2012) 336:1676-81. doi: $10.1126 /$ science. 1221063

16. Bousso P, Moreau HD. Functional immunoimaging: the revolution continues. Nat Rev Immunol. (2012) 12:858-64. doi: 10.1038/nri3342

17. Chong SZ, Evrard M, Goh CC, Ng LG. Illuminating the covert mission of mononuclear phagocytes in their regional niches. Curr Opin Immunol. (2018) 50:94-101. doi: 10.1016/j.coi.2017.12.004

\section{FUNDING}

This work was supported by the NMRC Young Individual Research grant (OFYIRG17may036) to SZC.

\section{ACKNOWLEDGMENTS}

We would like to thank Dr Akhila Balachander, Dr Adrian Boey, and Dr David Liebl for the scanning electron microscopy images of monocytes.

18. Hoeffel G, Ginhoux F. Fetal monocytes and the origins of tissue-resident macrophages. Cell Immunol. (2018) 330:5-15. doi: 10.1016/j.cellimm.2018.01.001

19. Palis J, Robertson S, Kennedy M, Wall C, Keller G. Development of erythroid and myeloid progenitors in the yolk sac and embryo proper of the mouse. Development. (1999) 126:5073-84.

20. Ferkowicz MJ, Yoder MC. Blood island formation: longstanding observations and modern interpretations. Exp Hematol. (2005) 33:1041-7. doi: 10.1016/j.exphem.2005.06.006

21. Takahashi K, Yamamura F, Naito M. Differentiation, maturation, and proliferation of macrophages in the mouse yolk sac: a light-microscopic, enzyme-cytochemical, immunohistochemical, and ultrastructural study. $J$ Leukoc Biol. (1989) 45:87-96. doi: 10.1002/jlb.45.2.87

22. Naito M, Yamamura F, Nishikawa S, Takahashi K. Development, differentiation, and maturation of fetal mouse yolk sac macrophages in cultures. J Leukoc Biol. (1989) 46:1-10. doi: 10.1002/jlb.46.1.1

23. Palis J, Chan RJ, Koniski A, Patel R, Starr M, Yoder MC. Spatial and temporal emergence of high proliferative potential hematopoietic precursors during murine embryogenesis. Proc Natl Acad Sci USA. (2001) 98:4528-33. doi: $10.1073 /$ pnas.071002398

24. McGrath KE, Koniski AD, Malik J, Palis J. Circulation is established in a stepwise pattern in the mammalian embryo. Blood. (2003) 101:1669-76. doi: 10.1182/blood-2002-08-2531

25. Gomez Perdiguero E, Klapproth K, Schulz C, Busch K, Azzoni E, Crozet L, et al. Tissue-resident macrophages originate from yolk-sac-derived erythromyeloid progenitors. Nature. (2015) 518:547-51. doi: 10.1038/nature13989

26. Hoeffel G, Chen J, Lavin Y, Low D, Almeida FF, See P, et al. C$\mathrm{Myb}(+)$ erythro-myeloid progenitor-derived fetal monocytes give rise to adult tissue-resident macrophages. Immunity. (2015) 42:665-78. doi: 10.1016/j.immuni.2015.03.011

27. Naito M, Takahashi K, Nishikawa S. Development, differentiation, and maturation of macrophages in the fetal mouse liver. J Leukoc Biol. (1990) 48:27-37. doi: 10.1002/jlb.48.1.27

28. Naito M, Umeda S, Yamamoto T, Moriyama H, Umezu H, Hasegawa G, et al. Development, differentiation, and phenotypic heterogeneity of murine tissue macrophages. J Leukoc Biol. (1996) 59:133-8. doi: 10.1002/jlb.59.2.133

29. Hoeffel G, Wang Y, Greter M, See P, Teo P, Malleret B, et al. Adult langerhans cells derive predominantly from embryonic fetal liver monocytes with a minor contribution of yolk sac-derived macrophages. J Exp Med. (2012) 209:1167-81. doi: 10.1084/jem.20120340

30. Guilliams M, De Kleer I, Henri S, Post S, Vanhoutte L, De Prijck S, et al. Alveolar macrophages develop from fetal monocytes that differentiate into long-lived cells in the first week of life via GM-CSF. J Exp Med. (2013) 210:1977-92. doi: 10.1084/jem.20131199

31. Schneider C, Nobs SP, Kurrer M, Rehrauer H, Thiele C, Kopf M. Induction of the nuclear receptor PPAR-gamma by the cytokine GM-CSF is critical for the differentiation of fetal monocytes into alveolar macrophages. Nat Immunol. (2014) 15:1026-37. doi: 10.1038/ni.3005

32. Epelman S, Lavine KJ, Beaudin AE, Sojka DK, Carrero JA, Calderon B, et al. Embryonic and adult-derived resident cardiac macrophages are maintained through distinct mechanisms at steady state and during inflammation. Immunity. (2014) 40:91-104. doi: 10.1016/j.immuni.2013.11.019 
33. Rantakari P, Jappinen N, Lokka E, Mokkala E, Gerke H, Peuhu E, et al. Fetal liver endothelium regulates the seeding of tissue-resident macrophages. Nature. (2016) 538:392-6. doi: 10.1038/nature19814

34. van de Laar L, Saelens W, De Prijck S, Martens L, Scott CL, Van Isterdael G, et al. Yolk sac macrophages, fetal liver, and adult monocytes can colonize an empty niche and develop into functional tissue-resident macrophages. Immunity. (2016) 44:755-68. doi: 10.1016/j.immuni.2016.02.017

35. Hettinger J, Richards DM, Hansson J, Barra MM, Joschko AC, Krijgsveld J, et al. Origin of monocytes and macrophages in a committed progenitor. Nat Immunol. (2013) 14:821-30. doi: 10.1038/ni.2638

36. Fogg DK, Sibon C, Miled C, Jung S, Aucouturier P, Littman DR, et al. A clonogenic bone marrow progenitor specific for macrophages and dendritic cells. Science. (2006) 311:83-7. doi: 10.1126/science.1117729

37. Chong SZ, Evrard M, Devi S, Chen J, Lim JY, See P, et al. CXCR4 identifies transitional bone marrow premonocytes that replenish the mature monocyte pool for peripheral responses. J Exp Med. (2016) 213:2293-314. doi: 10.1084 /jem.20160800

38. Terashima T, Wiggs B, English D, Hogg JC, van Eeden SF. Polymorphonuclear leukocyte transit times in bone marrow during streptococcal pneumonia. Am J Physiol. (1996) 271:L587-92. doi: 10.1152/ajplung.1996.271.4.L587

39. Goto Y, Hogg JC, Suwa T, Quinlan KB, van Eeden SF. A novel method to quantify the turnover and release of monocytes from the bone marrow using the thymidine analog 5'-bromo-2'-deoxyuridine. Am J Physiol Cell Physiol. (2003) 285:C253-9. doi: 10.1152/ajpcell.00035.2003

40. Tsou CL, Peters W, Si Y, Slaymaker S, Aslanian AM, Weisberg SP, et al. Critical roles for CCR2 and MCP-3 in monocyte mobilization from bone marrow and recruitment to inflammatory sites. J Clin Invest. (2007) 117:9029. doi: 10.1172/JCI29919

41. Serbina NV, Pamer EG. Monocyte emigration from bone marrow during bacterial infection requires signals mediated by chemokine receptor CCR2. Nat Immunol. (2006) 7:311-7. doi: 10.1038/ni1309

42. Jung H, Mithal DS, Park JE, Miller RJ. Localized CCR2 Activation in the bone marrow niche mobilizes monocytes by desensitizing CXCR4. PLoS ONE. (2015) 10:e0128387. doi: 10.1371/journal.pone.0128387

43. Liu Q, Li Z, Gao JL, Wan W, Ganesan S, McDermott DH, et al. CXCR4 antagonist AMD3100 redistributes leukocytes from primary immune organs to secondary immune organs, lung, and blood in mice. Eur J Immunol. (2015) 45:1855-67. doi: 10.1002/eji.201445245

44. Hamon P, Rodero MP, Combadiere C, Boissonnas A. Tracking mouse bone marrow monocytes in vivo. J Vis Exp. (2015) 96:e52476. doi: 10.3791/52476

45. Shi C, Jia T, Mendez-Ferrer S, Hohl TM, Serbina NV, Lipuma L, et al. Bone marrow mesenchymal stem and progenitor cells induce monocyte emigration in response to circulating toll-like receptor ligands. Immunity. (2011) 34:590601. doi: 10.1016/j.immuni.2011.02.016

46. Evrard M, Chong SZ, Devi S, Chew WK, Lee B, Poidinger M, et al. Visualization of bone marrow monocyte mobilization using Cx3cr1gfp/+Flt3L-/- reporter mouse by multiphoton intravital microscopy. J Leukoc Biol. (2015) 97:611-9. doi: 10.1189/jlb.1TA0514-274R

47. Jacquelin S, Licata F, Dorgham K, Hermand P, Poupel L, Guyon E, et al. CX3CR1 reduces Ly6Chigh-monocyte motility within and release from the bone marrow after chemotherapy in mice. Blood. (2013) 122:674-83. doi: 10.1182/blood-2013-01-480749

48. Debien E, Mayol K, Biajoux V, Daussy C, De Aguero MG, Taillardet M, et al. S1PR5 is pivotal for the homeostasis of patrolling monocytes. Eur J Immunol. (2013) 43:1667-75. doi: 10.1002/eji.201343312

49. Swirski FK, Nahrendorf M, Etzrodt M, Wildgruber M, Cortez-Retamozo $\mathrm{V}$, Panizzi $\mathrm{P}$, et al. Identification of splenic reservoir monocytes and their deployment to inflammatory sites. Science. (2009) 325:612-6. doi: $10.1126 /$ science. 1175202

50. Bronte V, Pittet MJ. The spleen in local and systemic regulation of immunity. Immunity. (2013) 39:806-18. doi: 10.1016/j.immuni.2013.10.010

51. Leuschner F, Rauch PJ, Ueno T, Gorbatov R, Marinelli B, Lee WW, et al. Rapid monocyte kinetics in acute myocardial infarction are sustained by extramedullary monocytopoiesis. J Exp Med. (2012) 209:123-37. doi: $10.1084 /$ jem. 20111009

52. Robbins CS, Chudnovskiy A, Rauch PJ, Figueiredo JL, Iwamoto Y, Gorbatov R, et al. Extramedullary hematopoiesis generates Ly-6C(high) monocytes that infiltrate atherosclerotic lesions. Circulation. (2012) 125:36474. doi: 10.1161/CIRCULATIONAHA.111.061986

53. Oakley OR, Kim H, El-Amouri I, Lin PC, Cho J, Bani-Ahmad M, et al. Periovulatory leukocyte infiltration in the rat ovary. Endocrinology. (2010) 151:4551-9. doi: 10.1210/en.2009-1444

54. Mellak S, Ait-Oufella H, Esposito B, Loyer X, Poirier M, Tedder TF, et al. Angiotensin II mobilizes spleen monocytes to promote the development of abdominal aortic aneurysm in Apoe-/- mice. Arterioscler Thromb Vasc Biol. (2015) 35:378-88. doi: 10.1161/ATVBAHA.114.304389

55. Wang NP, Erskine J, Zhang WW, Zheng RH, Zhang LH, Duron G, et al. Recruitment of macrophages from the spleen contributes to myocardial fibrosis and hypertension induced by angiotensin II. J Renin Angiotensin Aldosterone Syst. (2017) 18:1470320317706653. doi: $10.1177 / 1470320317706653$

56. Cortez-Retamozo V, Etzrodt M, Newton A, Ryan R, Pucci F, Sio SW, et al. Angiotensin II drives the production of tumor-promoting macrophages. Immunity. (2013) 38:296-308. doi: 10.1016/j.immuni.2012.10.015

57. Hsiao HM, Fernandez R, Tanaka S, Li W, Spahn JH, Chiu S, et al. Spleen-derived classical monocytes mediate lung ischemia-reperfusion injury through IL-1beta. J Clin Invest. (2018) 128:2833-47. doi: 10.1172/JCI98436

58. Varol C, Landsman L, Fogg DK, Greenshtein L, Gildor B, Margalit R, et al. Monocytes give rise to mucosal, but not splenic, conventional dendritic cells. J Exp Med. (2007) 204:171-80. doi: 10.1084/jem.20061011

59. Hanna RN, Carlin LM, Hubbeling HG, Nackiewicz D, Green AM, Punt JA, et al. The transcription factor NR4A1 (Nur77) controls bone marrow differentiation and the survival of Ly6C- monocytes. Nat Immunol. (2011) 12:778-85. doi: 10.1038/ni.2063

60. Yona S, Kim KW, Wolf Y, Mildner A, Varol D, Breker M, et al. Fate mapping reveals origins and dynamics of monocytes and tissue macrophages under homeostasis. Immunity. (2013) 38:79-91. doi: 10.1016/j.immuni.2012. 12.001

61. Patel AA, Zhang Y, Fullerton JN, Boelen L, Rongvaux A, Maini AA, et al. The fate and lifespan of human monocyte subsets in steady state and systemic inflammation. J Exp Med. (2017) 214:1913-23. doi: 10.1084/jem.20170355

62. Auffray C, Fogg D, Garfa M, Elain G, Join-Lambert O, Kayal S, et al. Monitoring of blood vessels and tissues by a population of monocytes with patrolling behavior. Science. (2007) 317:666-70. doi: 10.1126/science.1142883

63. Cros J, Cagnard N, Woollard K, Patey N, Zhang SY, Senechal B, et al. Human CD14dim monocytes patrol and sense nucleic acids and viruses via TLR7 and TLR8 receptors. Immunity. (2010) 33:375-86. doi: 10.1016/j.immuni.2010.08.012

64. Carlin LM, Stamatiades EG, Auffray C, Hanna RN, Glover L, VizcayBarrena G, et al. Nr4a1-dependent Ly6C(low) monocytes monitor endothelial cells and orchestrate their disposal. Cell. (2013) 153:362-75. doi: $10.1016 /$ j.cell.2013.03.010

65. Thomas G, Tacke R, Hedrick CC, Hanna RN. Nonclassical patrolling monocyte function in the vasculature. Arterioscler Thromb Vasc Biol. (2015) 35:1306-16. doi: 10.1161/ATVBAHA.114.304650

66. Quintar A, McArdle S, Wolf D, Marki A, Ehinger E, Vassallo M, et al. Endothelial protective monocyte patrolling in large arteries intensified by western diet and atherosclerosis. Circ Res. (2017) 120:1789-99. doi: 10.1161/CIRCRESAHA.117.310739

67. Michaud JP, Bellavance MA, Prefontaine P, Rivest S. Real-time in vivo imaging reveals the ability of monocytes to clear vascular amyloid beta. Cell Rep. (2013) 5:646-53. doi: 10.1016/j.celrep.2013.10.010

68. Hanna RN, Cekic C, Sag D, Tacke R, Thomas GD, Nowyhed H, et al. Patrolling monocytes control tumor metastasis to the lung. Science. (2015) 350:985-90. doi: 10.1126/science.aac9407

69. Finsterbusch M, Hall P, Li A, Devi S, Westhorpe CL, Kitching AR, et al. Patrolling monocytes promote intravascular neutrophil activation and glomerular injury in the acutely inflamed glomerulus. Proc Natl Acad Sci USA. (2016) 113:E5172-81. doi: 10.1073/pnas.1606253113

70. Rua R, McGavern DB. Elucidation of monocyte/macrophage dynamics and function by intravital imaging. J Leukoc Biol. (2015) 98:319-32. doi: 10.1189/jlb.4RI0115-006RR

71. Kuebler WM, Goetz AE. The marginated pool. Eur Surg Res. (2002) 34:92100. doi: $10.1159 / 000048894$ 
72. Rodero MP, Poupel L, Loyher PL, Hamon P, Licata F, Pessel C, et al. Immune surveillance of the lung by migrating tissue monocytes. Elife. (2015) 4:e07847. doi: 10.7554/eLife.07847

73. Looney MR, Thornton EE, Sen D, Lamm WJ, Glenny RW, Krummel MF. Stabilized imaging of immune surveillance in the mouse lung. Nat Methods. (2011) 8:91-6. doi: 10.1038/nmeth.1543

74. O'Dea KP, Wilson MR, Dokpesi JO, Wakabayashi K, Tatton L, van Rooijen $\mathrm{N}$, et al. Mobilization and margination of bone marrow Gr-1high monocytes during subclinical endotoxemia predisposes the lungs toward acute injury. $J$ Immunol. (2009) 182:1155-66. doi: 10.4049/jimmunol.182.2.1155

75. Devi S, Li A, Westhorpe CL, Lo CY, Abeynaike LD, Snelgrove SL, et al. Multiphoton imaging reveals a new leukocyte recruitment paradigm in the glomerulus. Nat Med. (2013) 19:107-12. doi: 10.1038/nm.3024

76. Chousterman BG, Boissonnas A, Poupel L, Baudesson de Chanville C, Adam J, Tabibzadeh N, et al. Ly6Chigh monocytes protect against kidney damage during sepsis via a CX3CR1-dependent adhesion mechanism. J Am Soc Nephrol. (2016) 27:792-803. doi: 10.1681/ASN.2015010009

77. Dal-Secco D, Wang J, Zeng Z, Kolaczkowska E, Wong CH, Petri B, et al. A dynamic spectrum of monocytes arising from the in situ reprogramming of CCR2+ monocytes at a site of sterile injury. J Exp Med. (2015) 212:447-56. doi: 10.1084/jem.20141539

78. Pai S, Qin J, Cavanagh L, Mitchell A, El-Assaad F, Jain R, et al. Real-time imaging reveals the dynamics of leukocyte behaviour during experimental cerebral malaria pathogenesis. PLoS Pathog. (2014) 10:e1004236. doi: 10.1371/journal.ppat.1004236

79. Hamon P, Loyher PL, Baudesson de Chanville C, Licata F, Combadiere C, Boissonnas A. CX3CR1-dependent endothelial margination modulates Ly6C(high) monocyte systemic deployment upon inflammation in mice. Blood. (2017) 129:1296-307. doi: 10.1182/blood-2016-08-732164

80. Nguyen KD, Fentress SJ, Qiu Y, Yun K, Cox JS, Chawla A. Circadian gene Bmal1 regulates diurnal oscillations of Ly6C(hi) inflammatory monocytes. Science. (2013) 341:1483-8. doi: 10.1126/science.1240636

81. Bain CC, Scott CL, Uronen-Hansson H, Gudjonsson S, Jansson O, Grip O, et al. Resident and pro-inflammatory macrophages in the colon represent alternative context-dependent fates of the same Ly6Chi monocyte precursors. Mucosal Immunol. (2013) 6:498-510. doi: 10.1038/mi.2012.89

82. Rivollier A, He J, Kole A, Valatas V, Kelsall BL. Inflammation switches the differentiation program of Ly6Chi monocytes from antiinflammatory macrophages to inflammatory dendritic cells in the colon. J Exp Med. (2012) 209:139-55. doi: 10.1084/jem.20101387

83. Nourshargh S, Alon R. Leukocyte migration into inflamed tissues. Immunity. (2014) 41:694-707. doi: 10.1016/j.immuni.2014.10.008

84. Stramer BM, Mori R, Martin P. The inflammation-fibrosis link? A Jekyll and Hyde role for blood cells during wound repair. J Invest Dermatol. (2007) 127:1009-17. doi: 10.1038/sj.jid.5700811

85. Patarroyo M, Prieto J, Beatty PG, Clark EA, Gahmberg CG. Adhesionmediating molecules of human monocytes. Cell Immunol. (1988) 113:278-89. doi: 10.1016/0008-8749(88)90027-5

86. Rodero MP, Licata F, Poupel L, Hamon P, Khosrotehrani K, Combadiere $\mathrm{C}$, et al. In vivo imaging reveals a pioneer wave of monocyte recruitment into mouse skin wounds. PLoS ONE. (2014) 9:e108212. doi: 10.1371/journal.pone.0108212

87. Goh CC, Evrard M, Chong SZ, Tan Y, Tan LL, Teng KWW, et al. The impact of ischemia-reperfusion injuries on skin resident murine dendritic cells. Eur J Immunol. (2018) 48:1014-9. doi: 10.1002/eji.2017 47347

88. Tamoutounour S, Henri S, Lelouard H, de Bovis B, de Haar C, van der Woude CJ, et al. CD64 distinguishes macrophages from dendritic cells in the gut and reveals the Th1-inducing role of mesenteric lymph node macrophages during colitis. Eur J Immunol. (2012) 42:3150-66. doi: 10.1002/eji.201242847

89. Serbina NV, Salazar-Mather TP, Biron CA, Kuziel WA, Pamer EG. TNF/iNOSProducing dendritic cells mediate innate immune defense against bacterial infection. Immunity. (2003) 19:59-70. doi: 10.1016/S1074-7613(03)00171-7

90. Rodero MP, Khosrotehrani K. Skin wound healing modulation by macrophages. Int J Clin Exp Pathol. (2010) 3:643-53.

91. Kumar V, Patel S, Tcyganov E, Gabrilovich DI. The nature of myeloid-derived suppressor cells in the tumor microenvironment. Trends Immunol. (2016) 37:208-20. doi: 10.1016/j.it.2016.01.004

92. Jakubzick C, Gautier EL, Gibbings SL, Sojka DK, Schlitzer A, Johnson TE, et al. Minimal differentiation of classical monocytes as they survey steady-state tissues and transport antigen to lymph nodes. Immunity. (2013) 39:599-610. doi: 10.1016/j.immuni.2013.08.007

93. Guilliams M, van de Laar L. A Hitchhiker's guide to myeloid cell subsets: practical implementation of a novel mononuclear phagocyte classification system. Front Immunol. (2015) 6:406. doi: 10.3389/fimmu.2015.00406

94. Tsujikawa T, Kumar S, Borkar RN, Azimi V, Thibault G, Chang YH, et al. Quantitative multiplex immunohistochemistry reveals myeloid-inflamed tumor-immune complexity associated with poor prognosis. Cell Rep. (2017) 19:203-17. doi: 10.1016/j.celrep.2017.03.037

95. Lin JR, Izar B, Wang S, Yapp C, Mei S, Shah PM, et al. Highly multiplexed immunofluorescence imaging of human tissues and tumors using t-CyCIF and conventional optical microscopes. Elife. (2018) 7:e31657. doi: 10.7554/eLife.31657

96. Gerner MY, Kastenmuller W, Ifrim I, Kabat J, Germain RN. Histo-cytometry: a method for highly multiplex quantitative tissue imaging analysis applied to dendritic cell subset microanatomy in lymph nodes. Immunity. (2012) 37:364-76. doi: 10.1016/j.immuni.2012.07.011

97. Gerner MY, Torabi-Parizi P, Germain RN. Strategically localized dendritic cells promote rapid $\mathrm{T}$ cell responses to lymph-borne particulate antigens. Immunity. (2015) 42:172-85. doi: 10.1016/j.immuni.2014.12.024

98. Li W, Germain RN, Gerner MY. Multiplex, quantitative cellular analysis in large tissue volumes with clearing-enhanced 3D microscopy (Ce3D). Proc Natl Acad Sci USA. (2017) 114:E7321-30. doi: 10.1073/pnas.1708981114

99. Tan Y, Li JLY, Goh CC, Lee BTK, Kwok IWH, Ng WJ, et al. Streamlining volumetric multi-channel image cytometry using huesaturation-brightness-based surface creation. Commun Biol. (2018) 1:136. doi: 10.1038/s42003-018-0139-y

Conflict of Interest Statement: The authors declare that the research was conducted in the absence of any commercial or financial relationships that could be construed as a potential conflict of interest.

Copyright (C) 2019 Teh, Ding, Ng and Chong. This is an open-access article distributed under the terms of the Creative Commons Attribution License (CC BY). The use, distribution or reproduction in other forums is permitted, provided the original author(s) and the copyright owner(s) are credited and that the original publication in this journal is cited, in accordance with accepted academic practice. No use, distribution or reproduction is permitted which does not comply with these terms. 